\title{
Parallel Isolation and Characterization of Porcine Smooth Muscle, Endothelial and Mesenchymal Stromal Cells for Bioengineering Applications
}

\section{Sara Morini}

Instituto de Investigación Sanitaria Aragón

Iris Pla-Palacín

Instituto de Investigación Sanitaria Aragón

Pilar Sainz-Arnal

Instituto de Investigación Sanitaria Aragón

Natalia Sánchez-Romero

Instituto de Investigación Sanitaria Aragón

Maria Falceto

University of Zaragoza

Olga Mitjana

University of Zaragoza

Antonio Romero

University of Zaragoza

Lucía Martinez-Santamaria

Instituto de Investigación Sanitaria Fundación Jiménez Díaz

Sara Guerrero-Aspizua

Instituto de Investigación Sanitaria Fundación Jiménez Díaz

Maria Bengochea Martinez

Instituto de Investigación Sanitaria Aragón

Emma Olmedo Arbizu

Instituto de Investigación Sanitaria Aragón

Ana Fernandes-Platzgummer

Department of Bioengineering and iBB - Institute for Bioengineering and Biosciences, Instituto Superior

Técnico, Universidade de Lisboa, Lisbon, Portugal

Pedro Baptista ( $\nabla$ pmbaptista@iisaragon.es )

Instituto de Investigación Sanitaria Aragón

Research Article 
Keywords: mesenchymal stromal cells, smooth muscle cells, endothelial cells, porcine, isolation, expansion, characterization

Posted Date: November 12th, 2021

DOI: https://doi.org/10.21203/rs.3.rs-960488/v1

License: (1) This work is licensed under a Creative Commons Attribution 4.0 International License. Read Full License 


\section{Abstract}

There is significant interest in the pig as the animal model of choice for organ transplantation and the study of tissue engineering (TE) products and applications. Currently, efforts are being taken to bioengineer solid organs to reduce donor shortages for transplantation. For complex organs such as the lung, heart, and liver, the vasculature represents a fundamental feature. Thus, to generate organs with a functional vascular network, the different cells constituting the building blocks of the blood vessels should be procured. However, due to species' specificities, porcine cell isolation, expansion, and characterization are not entirely straightforward compared to human cell procurement. Here, we report the establishment of simple and suitable methods for the isolation and characterization of distinct porcine cells for bioengineering purposes.

We successfully isolated, expanded and characterized porcine bone marrow-derived mesenchymal stromal (pBM-MSC), aortic smooth muscle (pASMC), and umbilical vein endothelial cells (pUVEC). We demonstrated that the three cell types showed specific immunophenotypical features. Moreover, we demonstrated that PBM-MSC could preserve their multipotency in vitro, and pUVEC were capable of maintaining their functionality in vitro.

These cultured cells could be further expanded and represent a useful cellular tool for TE purposes (i.e., for recellularization approaches of vascularized organs or in vitro angiogenesis studies).

\section{Introduction}

To date, organ transplantation represents the only available and definitive treatment for patients suffering from end-stage organ disease. This procedure effectively increases patient survival but is limited due to a dramatic shortage of organ donors [1]. This chronic scarcity of donated human organs for transplantation has motivated considerable interest in the production of biocompatible and fully functional bioengineered organs. This goal seems to be within reach through organ decellularization and recellularization technologies [2-4]. The resulting bioengineered organs can be additionally recellularized using other cell types and further matured to create more functional organs ready to be transplanted [5].

To restore the full function of an organ, it is fundamental that all components are correctly bioengineered since the overall function of an organ depends on the successful integration of its constituents (e.g., epithelia, mesoderm, parenchyma, and vasculature) [6-8]. The vasculature plays a crucial role as it represents the primary point of communication between each organ or tissue and the rest of the body. It is also vital to guarantee the delivery of oxygen and nutrients to the cells and remove cellular waste products. This aspect is critical in regenerative medicine approaches, especially for whole organ bioengineering, since oxygen delivery would be limited to a few hundred $\mu \mathrm{m}$ by simple gas diffusion in non-vascularized tissue [7]. This would undoubtedly result in cell/tissue necrosis, limiting the in vitro generation of organs and survival post-transplantation [8]. Moreover, EC play an active role in 
orchestrating the processes involved in tissue repair [9-15] and produce a non-thrombogenic barrier [16], which are critical features in the generation and engraftment procedures of bioengineered organs.

Thus, this study aimed to establish appropriate methods for the efficient and reproducible isolation, characterization, and expansion of distinct porcine cell types (EC, SMC, and MSC) in parallel for organ scaffold recellularization, as well as for other tissue engineering applications, i.e., in vitro angiogenesis studies. The rationale behind the use of porcine cells is due to the present and future usage of the pig as the animal model of choice for the transplantation of bioengineered livers [17]. Nevertheless, porcine and human cell procurement, expansion, and characterization are not precisely equivalent, and any developed procedures need to be extensively investigated and tested due to interspecies differences. Hence, in this work, we report practical and straightforward strategies for the parallel isolation of pure populations of cells from different biological samples. When expanded using the conditions described in this study, the isolated cells could actively proliferate, maintaining viability during the culture. Also, we identified a combination of appropriate antibodies and functional assays, allowing for a feasible characterization of the immunophenotypical and functional identity of these cells. Overall, considering the relevance of EC, SMC, and MSC in tissue engineering and regenerative medicine, and the usefulness of the pig as an animal model in this field, the methods reported herein may represent a highly suitable and straightforward tool for obtaining pure cultures of primary porcine cells with well-defined phenotypical and functional features for bioengineering applications, as showed in the experimental flowchart presented [Figure 1].

\section{Results}

\section{pBM-MSC isolation and culture}

Porcine BM MNC separation was achieved with Histopaque 1.077 gradient centrifugation. The MNC fraction was seeded with DMEM/FBS-MSC culture medium in tissue culture-treated $152 \mathrm{~cm}^{2}$ Petri dishes coated with $0.2 \%$ gelatin to improve cell adhesion. pBM-MSC adhered to the bottom of the dish and the non-adherent cells were removed during the first media change. Discrete cell colonies were evident at 3-5 days after the initial seeding and pBM-MSC population showed an elongated fibroblast-like appearance [Figure 2A], which is more evident in cells organized in colonies, as reported in the literature [20,21]. Cultures were heterogeneous and constituted by different subpopulations of cells, such as small polygonal cells interspersed with characteristic spindle-shaped cells [Figure 2B], as reported for human BM-MSC [22-24]. The number and size of the colonies increased progressively to reach confluence (80\%) 10-12 days after the initial seeding.

\section{Phenotypical and functional characterization of pBM-MSC}

The immunophenotypical features of pBM-MSC were determined by flow cytometry using a panel of surface markers, which have been described as representative of mesenchymal cell lineage (CD90), other antigens generally expressed by MSC (CD29, CD44), and lineage markers (CD11b, CD14, CD31, CD34, CD45). Essentially, all pBM-MSC expressed CD29, CD44, and CD90. However, the expression of 
endothelial marker CD31 and other markers present in hematopoietic cells like CD11b, CD14, CD34, and CD45 were absent [Figure 3A].

Furthermore, we assessed the expression of a set of surface markers (CD29, CD44, and CD90) by immunofluorescence [Figure 3B]. The cell staining result corroborated the data obtained by flow cytometry analysis. Thus, the data generated from both flow cytometry and immunofluorescence analysis allows us to state that PBM-MSC showed significant phenotypical features of MSC.

Moreover, we tested the trilineage differentiation capability of pBM-MSC into adipocytes, osteoblasts, and chondrocytes. This was confirmed by standard induction protocols using commercial differentiation kits. Adipogenic differentiation was evaluated by Oil Red $\mathrm{O}$ staining [Figure $4 \mathrm{~A}$ and D]. Some cells in the negative control with expansion medium could differentiate into adipocytes, even if to a relatively smaller extent than the cells treated with the adipogenic differentiation medium. This is a common effect in confluent cultures of MSC.

Nonetheless, Oil Red O staining revealed that pBM-MSC was capable of successfully differentiate to adipocytes. The osteogenic differentiation capability of pBM-MSC was evaluated by ALP/Von Kossa staining [Figure 4B and E]. The negative control, cultured in expansion medium, only showed a slight heterogeneous red staining induced by ALP [Figure 4B], while the cells treated with osteogenic differentiation medium significantly differentiated, as demonstrated by the presence of black stains indicating calcium deposits and more intense red staining which suggests the presence of osteogenic progenitor cells in the culture. Finally, the chondrogenic differentiation ability of pBM-MSC was determined by Alcian Blue staining. The negative control, cultured in expansion medium, did not show any sign of differentiation [Figure 4C], while the cells cultured with chondrogenic differentiation medium stained in dark blue, suggesting glycosaminoglycans' presence, a fundamental component of cartilage [Figure 4F].

Therefore, we demonstrated that pBM-MSC presented relevant mesenchymal features, including the expression of mesenchymal markers (CD29, CD44, and CD90) and the ability to differentiate into adipocytes, osteoblasts, and chondrocytes in vitro.

\section{pASMC isolation and culture}

Cells were isolated by enzymatic digestion of aortic tunica media after removing the endothelial cell fraction resident in the tunica intima, seeded in fibronectin-coated Petri dishes, and cultured in DMEM/FBS. The primary PASMC were attached to the culture dishes and distinguishable 48 hours after isolation [Figure 5A]. The selected pASMC population had a spindle-shaped fibroblast-like appearance [Figure 5B-D]. The number and size of the colonies increased progressively to reach confluence (90\%) 10 12 days after the initial seeding.

\section{Phenotypical characterization of pASMC}


To define the immunophenotypical features of pASMC, we evaluated by flow cytometry the expression of markers that have been described as representative of smooth muscle cells (SM22 $\alpha$ ) [25], more mesenchymal-like (CD90), other antigens generally expressed by MSC (CD29, CD44), a muscular marker also described in a subset of MSC (CD56) and lineage markers (CD11b, CD14, CD31, CD34, CD45, CD144). Essentially, all pASMC expressed SM22 $\alpha$, CD29, CD44, CD56, and CD90. However, the expression of the endothelial markers CD31 and CD144, as well as the lineage markers CD11b, CD14, CD34, and CD45, was absent [Figure 6A].

Moreover, we assessed the expression of a panel of intracellular and extracellular markers, including smooth muscle markers (SM22 $\alpha, \alpha$ SMA, and Caldesmon) and other antigens commonly expressed by SMC (CD29, CD44, CD90) by immunofluorescence analysis. All the tested markers were highly expressed by pASMC [Figure 6B]. Notably, the most expressed markers were the contractile SMC markers SM22 $\alpha$ and $\alpha \mathrm{SMA}$, reported as contractile phenotype-associated markers in SMC [25].

Thus, considering the data generated from both flow cytometry and immunofluorescence analysis, we can state that the isolated SMC from the porcine aorta showed phenotypical features characteristic of SMC.

\section{pUVEC isolation and culture}

The cells were isolated by enzymatic digestion, seeded in $1 \%(\mathrm{v} / \mathrm{v})$ gelatin-coated Petri dishes, and cultured in a porcine endothelial growth medium (pEGM). The primary pUVEC were selected by their adhesive properties. Cell cultures were heterogeneous and constituted of different subpopulations of cells, including cobblestone-like shaped cells and elongated cells. To remove the contaminant cells from the culture we selected the CD31-positive cell fraction by cell sorting [Figure 7]. This allowed to obtain pure cultures of pUVEC which have been used for further experiments.

\section{Phenotypical and functional characterization of pUVEC}

To define the immunophenotypical profile of PUVEC, we evaluated the expression of markers that have been described as representative endothelial (CD31, CD105, CD144) [26], a progenitor marker (CD34), and mesenchymal cell markers (CD29, CD44) by flow cytometry. Essentially, all pUVEC expressed CD29, CD31, CD44, CD105, and CD144, with lack of expression of CD34 [Figure 8A].

Moreover, we assessed the expression of endothelial markers (CD31, CD105, CD144, Tie-2), other antigens commonly expressed by EC (CD44), and vimentin, an intermediate filament of the cytoskeleton which plays a critical role in the physiological endothelial mechano-response and inherent to the endothelial phenotype $[27,28]$. Notably, the most common markers for EC (CD31, CD105, CD144, and Tie2) were highly expressed by pUVEC, as well as Vimentin [Figure 8B].

To further demonstrate the endothelial phenotype of pUVEC, we tested the functionality of the cultured cells with the Dil-Ac-LDL uptake assay. Indeed EC, but not SMC, can internalize and metabolize Ac-LDL, which is detectable using a labeled form of the molecule, the probe $1,1^{\prime}$-dioctadecyl-3,3,3',3'-tetramethyl- 
indocarbocyanine perchlorate (Dil), as reported in the literature [29]. The Dil-Ac-LDL uptake assay showed that pUVEC significantly internalized the dye, as demonstrated by the associated red fluorescence detectable using a conventional fluorescence microscope [Figure 9].

Therefore, considering the results obtained from flow cytometry, immunofluorescence analysis, and the functional assay, we demonstrated that pUVEC isolated from porcine umbilical vein showed a robust endothelial phenotype.

\section{Discussion}

To date, the only definitive treatment for end-stage organ disease is orthotopic transplantation. Conventional surgical practices typically utilize organs from living or deceased donors for transplantation. However, the deficit between the number of patients waiting for a life-saving transplant and the number of organs available for transplantation has grown substantially in recent years. As a result, new options such as tissue engineering and regenerative medicine are gaining traction to overcome organ shortage.

Decellularization is a promising new approach in regenerative medicine that allows researchers to generate three-dimensional biological scaffolds by selective removal of all the cellular and nuclear components of an organ without significantly modifying the architecture and biochemistry of the vasculature, which can be used as an easy route to deliver cells throughout the entire organ $[2,30,31]$. Complete recellularization of the whole vascular network of an acellular scaffold represents a huge bottleneck towards the translation of bioengineered organs to the clinic. Blood vessels, such as arteries and veins, consist of three layers: tunica intima, mainly composed of EC; tunica media, which consists of connective tissue and SMC; and tunica adventitia, composed mainly of connective tissue. Capillaries and sinusoids are composed of a single layer of flattened EC and a basal lamina with few pericytes [32]. Therefore, EC, SMC, and pericytes/MSC must be considered fundamental bricks to rebuild the vasculature while using the preserved native architecture of the acellular organ as a guiding blueprint. In this regard, and to thoroughly investigate this, the pig has been consistently used in the past years as one of the animal models of choice for regenerative medicine and transplantation research [17,33]. Thus, isolating porcine vascular cells for future organ bioengineering applications is paramount to advancing the field without increasing the experimental complexity by crossing xenogeneic immune barriers using human cells transplanted in pigs [33].

Hence, to define optimal cell sources for organ revascularization, we isolated the three main cellular components of porcine blood vessels, expanded under conventional bidimensional culture conditions, and characterized them by their immunophenotype and functionality. We found that all the three cell types isolated and cultured could maintain specific phenotypical features, as demonstrated by flow cytometry and immunofluorescence analysis and cell functionality, as shown through pBM-MSC trilineage differentiation and pUVEC Dil-Ac-LDL uptake assays. Additionally, we confirmed that our culture medium containing 10\% FBS MSC Qualified induced robust cell proliferation providing adequate support 
for pBM-MSC expansion [34]. However, it is essential to highlight that there is still a lack of consensus regarding the definition of the optimal markers and methods to verify the identity of pBM-MSC $[21,35-$ 37]. Thus, considering the existing literature, we tested a panel of markers to determine the phenotype of pBM-MSC. Many molecules involved in cell adhesion and ECM proteins, cytokines, and growth factor receptors are expressed by MSC and are all associated with their functions and cell interactions within the bone marrow $[38,39]$. We showed that cultured pBM-MSC were adherent to plastic, with characteristic spindle-shaped morphology and expressed specific mesenchymal surface markers (CD90, CD29, and CD44), while they did not express lineage markers (CD11b, CD14, CD31, CD34, CD45), as reported in the literature [21,40-43]. A general lack of consensus also exists about the optimal differentiation conditions for pBM-MSC [21]. Thus, we performed in vitro differentiation of pBM-MSC into adipocytes, osteoblasts, and chondrocytes using commercial differentiation kits to obtain further information regarding the functionality of pBM-MSC. As these kits are formulated for human MSC, we followed the manufacturer's instructions, and we differentiated the porcine cells for 21 days. The multipotency of pBM-MSC has been demonstrated, as the cells could differentiate into the three lineages, confirming other reported results $[20,21,34,40]$.

Moreover, it has been described that adipogenic differentiation kinetics of pBM-MSC are different from human MSC, and fat accumulates initially in the adipocytes but is secreted afterward. This behavior is observed in pBM-MSC but not in human MSC. Therefore, the measurement of adipogenic differentiation by Oil Red $O$ staining may underestimate the actual fat formed by pBM-MSC [21]. From our results, we could show that pBM-MSC can be successfully differentiated using these human MSC formulated commercial kits.

Vascular SMC are present in distinct phenotypic states in blood vessels. Indeed, they exhibit different morphological and functional properties within the same blood vessel $[44,45]$ and different blood vessels, e.g., arteries and veins [46]. SMC show a high degree of plasticity and the ability to switch from a contractile to a synthetic phenotype in response to environmental stresses and vascular injury. This plasticity creates a complex diversity among SMC and is associated with changes in morphology, proliferation and migration rates, and the expression of different marker proteins. Contractile SMC express SM22 $\alpha, \alpha$ SMA, Caldesmon, smooth muscle calponin, smooth muscle myosin heavy chain, and smoothelin, among other markers, while synthetic SMC express osteopontin, collagen I, moesin, plateletderived growth factor $A$ and other markers [25]. Cell morphology represents an essential parameter for the definition of SMC phenotype. Contractile SMC are elongated, spindle-shaped cells, while synthetic SMC are epithelioid or rhomboid with a cobblestone-like morphology $[47,48]$. In this study, we have efficiently isolated vascular SMC from the porcine aorta, which retained important phenotypic features of contractile SMC when in culture. Indeed, pASMC showed a characteristic spindle-shaped morphology and expressed specific muscle markers (SM22 $\alpha, \alpha$ SMA, Caldesmon) and mesenchymal markers (CD90, CD29, CD44) while the expression of lineage markers were absent (CD11b, CD14, CD31, CD34, CD45, CD144). In addition, the cells expressed CD56, which is considered a natural killer, neuronal, and muscle marker but has also been described as a marker of a subset of MSC derived from human bone marrow [49-51]. $\alpha$ SMA is the most general marker of SMC lineage, and it is highly expressed in spindle-shaped 
SMC, while SM22 $\alpha$, smoothelin, calponin, and Caldesmon are late differentiation markers abundantly expressed in spindle-shaped SMC [52-54]. CD44 is a cell-surface receptor of hyaluronate, which has been described on various cell types that play a role in ECM binding, cell adhesion, cell-cell interactions, and cell migration $[55,56]$. The expression of this mesenchymal marker is reported in vascular cells derived from the porcine aorta [57] and rat aortic SMC [58]. Also, our results are consistent with the results showed by Zaniboni et al. that reported that vascular cells derived from the tunica media of porcine aorta expressed CD56 and CD90, while conversely, the cells did not express the hematopoietic markers CD34 and CD45 [57]. Our results show that pASMC express CD29 is also consistent with several published reports, demonstrating that integrin beta 1 (i.e., CD29) is predominant in vascular SMC in vivo and cultured SMC $[59,60]$. Therefore, we have shown that pASMC isolated from the aorta's tunica media showed specific mesenchymal surface markers. Moreover, the cells exhibited an elongated, fusiform, spindle-shaped morphology and expressed CD29, SM22 $\alpha, \alpha$ SMA, and Caldesmon, which are marker proteins of the contractile phenotype of SMC.

$\mathrm{EC}$ are the main component of the endothelium, which serves as a permeable barrier for blood vessels and regulates blood flow. EC are present in the whole vascular system, from the heart to capillaries, and control the exchanges of materials and the transit of white blood cells in and out of the bloodstream. Furthermore, EC are involved in regulating blood flow, coagulation, endothelial permeability, vascular tone, and vascular remodeling in responses to physiological and pathological stimuli. In terms of recellularization of decellularized scaffolds, a complete re-endothelialization of the acellular vascular network is required to avoid thrombosis after transplantation, induced by the interaction between ECM proteins and the plasma clotting factors and platelets. In this study, we were able to isolate vascular EC from the porcine umbilical vein and expand them under conventional bidimensional culture conditions. The isolated primary cultures were composed of heterogeneous cell populations with different morphology, similar to the morphology reported for their human equivalents [61] and Chrusciel and colleagues' work [62]. After that, we characterized the cells by flow cytometry and immunofluorescence analysis to define the phenotypical identity of expanded pUVEC. We showed that pUVEC expressed specific endothelial markers (CD31, CD105, CD144, Tie-2) and mesenchymal markers (CD29, CD44, Vimentin), while the expression of CD34 was absent. The expression of endothelial markers such as CD31, CD105, and CD144 has been previously reported for EC isolated from the porcine umbilical vein [26,62], aorta [18,63], heart [64], as well as for EC isolated from the human umbilical vein [65].

Moreover, the expression of vimentin, an intermediate filament of the cytoskeleton which plays a critical role in the physiological endothelial mechano-response and inherent to the endothelial phenotype [27,28], has been reported for human umbilical vein EC [66]. Further, we assessed the functionality of pUVEC by Dil-Ac-LDL uptake assay and showed that cultured pUVEC were able to internalize the fluorescent-labeledDil-Ac-LDL with success. These results suggest that pUVEC could maintain specific endothelial immunophenotypical features and functionality when isolated and cultured under these defined conditions. 


\section{Conclusions}

Our experiments demonstrated that pBM-MSC, pASMC, and pUVEC are MSC, SMC, and EC, as evidenced by their immunophenotypical features and functionality. Like human MSC, pBM-MSC are plasticadherent, express CD90, CD29, and CD44, and do not express lineage markers (CD11b, CD14, CD31, CD34, CD45). As evidenced by trilineage differentiation assay, pBM-MSC can successfully differentiate into adipocytes, osteoblasts, and chondrocytes. Moreover, like human contractile SMC, pASMC isolated from aorta's tunica media are spindle-shaped, with specific "hill and valley" morphology, and expressed a panel of markers associated with the differentiated contractile state of SMC (SM22 $\alpha, \alpha$ SMA, and Caldesmon) as well as mesenchymal-associated markers (CD90, CD29, CD44, CD56) whereas they do not express lineage markers (CD11b, CD14, CD31, CD34, CD45, CD144). Furthermore, like human umbilical vein EC, pUVEC express several endothelial markers (CD31, CD105, CD144, Tie-2) and mesenchymal markers (CD29, CD44, Vimentin), while they do not express CD34. As shown by the Dil-AcLDL uptake assay, pUVEC can successfully internalize the fluorescent dye, a clear indication of EC functionality.

In conclusion, this study provides suitable and efficient methods for the parallel isolation of pBM-MSC, pASMC, and pUVEC and convenient and straightforward cell culture procedures for cell expansion under conventional bidimensional conditions valuable and rigorous characterization of the cultured porcine cells.

Overall, our results showed that it is possible to isolate, expand and characterize pBM-MSC, pASMC, and pUVEC successfully using our reported methods. Thus, the obtained cells could be further expanded and could represent a valuable cellular tool for tissue engineering purposes, i.e., for recellularization approaches of any vascularized organ or for angiogenesis studies using co-culture assays to understand cell interactions under physiological conditions in bidimensional and three-dimensional cultures.

\section{Methods}

\section{Animal tissues}

Bone marrow and aorta tissues were obtained from male and female domestic piglets weighing $5-7 \mathrm{~kg}$. All animals $(n=4)$ were housed at the Experimental Surgery Department under approved conditions and the tissues were harvested after intraoperative approved euthanasia. Umbilical cords were obtained from pig fetuses ( $n=12,72-80$ days after insemination) after a planned cesarean section performed under hospital conditions at the Veterinary School of the University of Zaragoza. The experiments were performed in accordance with the European and ARRIVE guidelines regarding the protection of animals and were approved by the Animal Experimentation Committee of the Government of Aragón, Spain (ref. PI08/17).

\section{Isolation and cultivation of pBM-MSC}


Bone marrow was harvested under aseptic conditions from the femur of sacrificed and bled piglets (57Kg). Bone-marrow mononuclear cells (MNC) were separated with a Histopaque density gradient (1.077 $\mathrm{g} / \mathrm{ml}$ ) (Sigma-Aldrich, Merck, Germany) and washed twice with phosphate-buffered saline solution (PBS) (Sigma-Aldrich, Merck, Germany). Then, the pellet was centrifuged and resuspended in Dulbecco's Modified Eagle Medium (DMEM) (Gibco, ThermoFisher Scientific, USA) + 10\% (v/v) Fetal Bovine Serum MSC Qualified (FBS) (Gibco, ThermoFisher Scientific, USA) + 1\% (v/v) Penicillin/Streptomycin 100X (P/S) (Gibco, ThermoFisher Scientific, USA) (DMEM/FBS-MSC). MNC were seeded between 200,000 and 500,000 cells $/ \mathrm{cm}^{2}$ in $152 \mathrm{~cm}^{2}$ Petri dishes (Corning, USA) coated with $0.2 \%$ (v/v) gelatin (Sigma-Aldrich, Merck, Germany) at $37^{\circ} \mathrm{C}$ in a humidified atmosphere of $5 \% \mathrm{CO}_{2}$. Once confluent (80\%), cells were either frozen or sub-cultured following cell detachment with $0.05 \%(\mathrm{v} / \mathrm{v})$ trypsin $/ 0.02 \%(\mathrm{v} / \mathrm{v})$ EDTA (Gibco, ThermoFisher Scientific, USA) and trypsin inactivation with DMEM $+10 \%(\mathrm{v} / \mathrm{v})$ FBS (Gibco, ThermoFisher Scientific, USA) $+1 \%(\mathrm{v} / \mathrm{v}) \mathrm{P} / \mathrm{S}$ (DMEM/FBS) and seeded at $3,000 \mathrm{cells} / \mathrm{cm}^{2}$ in $152 \mathrm{~cm}^{2}$ Petri dishes coated with $0.2 \%(\mathrm{v} / \mathrm{v})$ gelatin. Passage $3-5$ cells were used in experiments. The photos were captured with an inverted optical microscope (Leica DMI3000B/Nikon Digital Camera Dxm1200F).

\section{Isolation and cultivation of pASMC}

The porcine aorta was procured from 5-7 kg piglets. pASMC were isolated using a modified version of the protocol published by Beigi and colleagues [18]. In a laminar flow hood and sterile conditions, the aorta was rinsed with cold calcium, and magnesium-free PBS supplemented with $1 \%(\mathrm{v} / \mathrm{v}) \mathrm{P} / \mathrm{S}$ to remove all residual blood. The aortic tube was dissected to eliminate the external elastic lamina and remove the tunica adventitia. One extremity of the vessel was ligated with a surgical 2-0 silk suture (B. Braun Melsungen, Germany) and checked for leaks with PBS. Next, the aorta was filled with dispase II protease (Sigma-Aldrich, Merck, Germany) (2,5 U/ml in DMEM) supplemented with $1 \%(\mathrm{v} / \mathrm{v}) \mathrm{P} / \mathrm{S}$, and the other end of the tube was ligated. The resulting vessel was incubated for 1 hour at $37^{\circ} \mathrm{C}$ in a humidified atmosphere of $5 \% \mathrm{CO}_{2}$. Then, the enzyme solution from the aorta was collected using an equal volume of

DMEM/FBS, and removed to eliminate the endothelial cell fraction of the aorta. After this, the tissue was cut open and placed in a Petri dish with the tunica intima facing up and scraped away any remaining EC using a cell scraper (ThermoFisher Scientific, USA). The tunica media layer was cut into 1-2 mm pieces, placed in a $50 \mathrm{ml}$ conical centrifuge tube (Corning, USA), and incubated for 1 to 1.5 hours in $0.2 \%(\mathrm{w} / \mathrm{v})$ collagenase type I (Sigma-Aldrich, Merck, Germany) in DMEM $+1 \%(\mathrm{v} / \mathrm{v}) \mathrm{P} / \mathrm{S}$ at $37^{\circ} \mathrm{C}$ with agitation at $210 \mathrm{rpm}$ on an orbital shaker (Innova 40, New Brunswick Scientific, USA). This collagenase solution was then discarded and replaced with $0.1 \%(\mathrm{w} / \mathrm{v})$ collagenase type I in DMEM $+1 \% \mathrm{P} / \mathrm{S}$ and incubated for 5 hours at $37^{\circ} \mathrm{C}$ with agitation at $230 \mathrm{rpm}$ on an orbital shaker (Innova 40, New Brunswick Scientific, USA). After 5 hours, the cell-enzyme solution was deactivated with an equal volume of DMEM/FBS and filtered through a $100 \mu \mathrm{m}$ and then a $40 \mu \mathrm{m}$ cell strainer (Corning, USA) to eliminate large pieces of undigested aortic tissue. The collected cell suspension was centrifuged, and the pellet was resuspended in DMEM/FBS. The obtained pASMC were seeded at 6,000-14,000 cells/ $\mathrm{cm}^{2}$ depending on the yield of isolated cells in $152 \mathrm{~cm}^{2}$ Petri dishes coated with $5 \mu \mathrm{g} / \mathrm{ml}$ fibronectin (Sigma-Aldrich, Merck, Germany) in DMEM/FBS at $37^{\circ} \mathrm{C}$ in a humidified atmosphere of $5 \% \mathrm{CO}_{2}$. The medium was replaced with a fresh 
medium after 48 hours from the initial seeding and changed every three days. Once confluent (90\%), cells were either frozen or sub-cultured following cell detachment with $0.05 \%(\mathrm{v} / \mathrm{v})$ trypsin/0.02\% (v/v) EDTA and trypsin inactivation with DMEM/FBS and seeded at 6,000 cells $/ \mathrm{cm}^{2}$ in $152 \mathrm{~cm}^{2}$ Petri dishes coated with $0.2 \%(\mathrm{v} / \mathrm{v})$ gelatin in DMEM/FBS and let at $37^{\circ} \mathrm{C}$ in a humidified atmosphere of $5 \% \mathrm{CO}_{2}$. Passage $3-5$ cells were used in experiments. The photos were captured with an inverted optical microscope (Leica DMI3000B/Nikon Digital Camera Dxm1200F).

\section{Isolation and cultivation of pUVEC}

Porcine umbilical cords were procured from swine fetuses at 72-80 days post-insemination. The cells were isolated from the umbilical vein by enzymatic digestion with $0.05 \%$ trypsin/ $0.02 \%$ EDTA, following an adapted protocol of Davis and co-workers [19]. After isolation, the cells were seeded at 15,000 cells $/ \mathrm{cm}^{2}$ in $152 \mathrm{~cm}^{2}$ Petri dishes coated with $1 \%(\mathrm{v} / \mathrm{v})$ gelatin in porcine endothelial growth medium (pEGM) (Cell Applications, USA) and incubated at $37^{\circ} \mathrm{C}$ in a humidified atmosphere of $5 \% \mathrm{CO}_{2}$. The medium was replaced with a fresh medium after 48 hours from the initial seeding and changed every two days. Once confluent $(100 \%)$, cells were either frozen or sub-cultured following cell detachment with $0.05 \%$ trypsin/0.02\% EDTA and trypsin inactivation with DMEM/FBS and seeded between 3,000 and 6,000 cells $/ \mathrm{cm}^{2}$ in $152 \mathrm{~cm}^{2}$ Petri dishes coated with $1 \%(\mathrm{v} / \mathrm{v})$ gelatin with $\mathrm{pEGM}$ and incubated at $37^{\circ} \mathrm{C}$ in a humidified atmosphere of $5 \% \mathrm{CO}_{2}$. The medium was changed every 5 days. To remove any contaminant cells from the culture, the cells were selected by cell sorting. Briefly, the cells were harvested, pelleted, counted, resuspended in basic flow cytometry buffer (FC Buffer: $2 \%$ FBS in PBS) and incubated with phycoerythrin (PE) conjugated antibody anti-CD31 (Clone: LCl-4, Bio-Rad, USA). The cells were washed three times with FC Buffer, centrifugated and resuspended in basic sorting buffer (1\% Bovine Serum Albumin (BSA) (Linus, Cultek, Spain) + 1mM EDTA in PBS + 1\% P/S) for the following cell separation. Cell sorting was carried out using a BD FACSJazz cell sorter (Becton Dickinson, USA). Then, the cells were seeded between 3,000 and 6,000 cells $/ \mathrm{cm}^{2}$ in $152 \mathrm{~cm}^{2}$ Petri dishes coated with $1 \%$ gelatin with pEGM and let at $37^{\circ} \mathrm{C}$ in a humidified atmosphere of $5 \% \mathrm{CO}_{2}$. The medium was changed every 5 days. Generally, passage 5-8 cells were used in the experiments. The pictures were captured with an inverted optical microscope (Leica DMI3000B/Nikon Digital Camera Dxm1200F).

\section{Phenotypical characterization of pBM-MSC, pASMC and pUVEC}

Flow cytometry and immunofluorescence assay were used to assess the expression of a panel of specific extracellular and intracellular markers of pBM-MSC, pASMC, and pUVEC. The list of antibodies used for cell characterization is shown in Table 1. A minimum of 10,000 events were collected for flow cytometry, and cells were analyzed on a BD FACSCalibur flow cytometer using Cell Quest Software (Becton Dickinson, USA). All data were analyzed using FlowJo Software (FlowJo, Becton Dickinson, USA).

The flow cytometry data of pBM-MSC, pASMC, and pUVEC was confirmed by immunofluorescence staining in 24-wells plates (ThermoFisher Scientific, USA). The cells were cultured under static conditions 
in an expansion medium at $37^{\circ} \mathrm{C}$ in a humidified atmosphere of $5 \% \mathrm{CO}_{2}$. pBM-MSC were seeded at 3,000 cells $/ \mathrm{cm}^{2}$ in 24 -wells plates coated with $0.2 \%$ gelatin, and the expansion medium was changed every 5 days until reaching confluence (80-90\%). pASMC were seeded at 6,000 cells $/ \mathrm{cm}^{2}$ in 24 -wells plates coated with $0.2 \%(\mathrm{v} / \mathrm{v})$ gelatin at $37^{\circ} \mathrm{C}$, and the expansion medium was changed every 5 days until reaching confluence (80-90\%). Finally, pUVEC were seeded at 4,000 cells $/ \mathrm{cm}^{2}$ in 24 -wells plates coated with $1 \%(\mathrm{v} / \mathrm{v})$ gelatin, and the expansion medium was changed every 5 days until reaching confluence $(100 \%)$. After fixation with $4 \%(\mathrm{v} / \mathrm{v})$ paraformaldehyde for 15 minutes, the cells were immunostained with several primary antibodies (Table 1 ) and an appropriate secondary antibody. The photos were captured with a fluorescence microscope (Leica DMI3000B/Nikon Digital Camera Dxm1200F) and processed using ImageJ 1.51s software. 
Table 1

List of the distinct antibodies used for the immunophenotypical characterization of pBM-MSC, pASMC, and PUVEC.

\begin{tabular}{|c|c|c|}
\hline Cell type & Analytical method & Antibodies \\
\hline \multirow{13}{*}{ pBM- } & \multirow[t]{9}{*}{ Flow Cytometry } & Primary Antibodies \\
\hline & & FITC conjugated anti-CD11b (Clone: ICRF44, Biolegend, USA); \\
\hline & & FITC conjugated anti-CD45 (Clone: K252.1E4, Bio-Rad, USA); \\
\hline & & PE-conjugated anti-CD14 (Clone: M5E2, Biolegend, USA); \\
\hline & & PE-conjugated anti-CD29 (Clone: TS2/16, Biolengend, USA); \\
\hline & & PE-conjugated anti-CD31 (Clone: LCl-4 Bio-Rad, USA); \\
\hline & & $\begin{array}{l}\text { Alexa Fluor } 488 \text { conjugated anti-CD } 44 \text { (Clone: IM7, Biolegend, } \\
\text { USA); }\end{array}$ \\
\hline & & $\begin{array}{l}\text { PerCP-Cy } 5.5 \text { conjugated anti-CD90 (Clone: 5E10, Biolegend, } \\
\text { USA); }\end{array}$ \\
\hline & & Anti-CD34 (Polyclonal, bs-8996R, Bioss Antibodies, USA) \\
\hline & \multirow[t]{4}{*}{ Immunofluorescence } & Primary Antibodies \\
\hline & & PE-conjugated anti-CD29 (Clone: TS2/16, Biolengend, USA); \\
\hline & & Anti-CD44 (Clone: IM7, StemCell Technologies, UK); \\
\hline & & Anti-CD90 (Clone: 5E10, StemCell Technologies, UK) \\
\hline \multirow[t]{10}{*}{ pASMC } & \multirow[t]{5}{*}{ Flow Cytometry } & Primary Antibodies \\
\hline & & Same as pBM-MSC, except: \\
\hline & & APC conjugated anti-CD56 (Clone: HCD56, Biolegend, USA); \\
\hline & & Anti-CD144 (Clone: F-8, Santa Cruz Biotehnology, USA); \\
\hline & & $\begin{array}{l}\text { Anti-SM22a (Clone: TAGLN/247, Novus-Biologicals, Bio-Techne, } \\
\text { UK) }\end{array}$ \\
\hline & \multirow[t]{5}{*}{ Immunofluorescence } & Primary Antibodies \\
\hline & & Same as pBM-MSC, except: \\
\hline & & $\begin{array}{l}\text { Anti-SM22a (Clone: TAGLN/247, Novus-Biologicals, Bio-Techne, } \\
\text { UK); }\end{array}$ \\
\hline & & Anti- aSMA (Clone: SP171, Sigma-Aldrich, Germany); \\
\hline & & Caldesmon (Clone: C21, Santa Cruz Biotechnology, USA) \\
\hline
\end{tabular}




\begin{tabular}{|c|c|c|}
\hline Cell type & Analytical method & Antibodies \\
\hline \multirow[t]{14}{*}{ PUVEC } & Flow Cytometry & Primary Antibodies \\
\hline & & PE conjugated anti-CD29 (Clone: Ts/16, Biolegend, USA); \\
\hline & & PE conjugated ant-CD31 (Clone: LCl-4, Bio-Rad, USA); \\
\hline & & $\begin{array}{l}\text { Alexa Fluor } 488 \text { conjugated anti-CD } 44 \text { (Clone: IM7, Biolegend, } \\
\text { USA); }\end{array}$ \\
\hline & & Anti-CD34 (Polyclonal, bs-8996R, Bioss Antibodies, USA); \\
\hline & & $\begin{array}{l}\text { Anti-CD105 (Clone: MEM-229, Novus-Biologicals, Bio-Techne, } \\
\text { UK); }\end{array}$ \\
\hline & & Anti-CD144 (Clone: F-8, Santa Cruz Biotechnology, USA) \\
\hline & Immunofluorescence & Primary Antibodies \\
\hline & & Anti-CD31 (Clone: H-3, Bioss Antibodies, USA); \\
\hline & & Anti-CD44 (Clone: IM7, StemCell Technologies, UK); \\
\hline & & $\begin{array}{l}\text { Anti-CD105 (Clone: MEM-229, Novus-Biologicals, Bio-Techne, } \\
\text { UK); }\end{array}$ \\
\hline & & Anti-CD144 (Clone: F-8, Santa Cruz Biotechnology, USA); \\
\hline & & Anti-Tie-2 (Clone: C-20, Santa Cruz Biotechnology, USA); \\
\hline & & Anti-Vimentin (Clone: V9, Santa Cruz Biotechnology, USA) \\
\hline
\end{tabular}

\section{Functional characterization of pBM-MSC}

To further characterize pBM-MSC, we evaluated the trilineage differentiation potential of these cells using commercial differentiation kits (StemPro Adipogenesis Differentiation Kit, StemPro Osteogenesis Differentiation Kit, StemPro Chondrogenesis Differentiation Kit, all from ThermoFisher Scientific, USA). Briefly, for adipogenic and osteogenic differentiation assays the cells were cultured in 24-wells plates coated with $0.2 \%(\mathrm{v} / \mathrm{v})$ gelatin at $10,000 \mathrm{cells} / \mathrm{cm}^{2}$ in expansion medium (DMEM/FBS) until confluence $(80 \%)$ at $37^{\circ} \mathrm{C}$ in a humidified atmosphere of $5 \% \mathrm{CO}_{2}$. Then, the expansion medium was replaced with a differentiation medium, which was changed every 3 days for 21 days. The differentiation was assessed by Oil Red O (for adipogenic differentiation) and alkaline phosphatase (ALP)/Von Kossa (for osteogenic differentiation) stainings. The cells were harvested, centrifuged, counted, and resuspended in expansion medium for the chondrogenic differentiation assay to generate hanging drops of cells for differentiation.

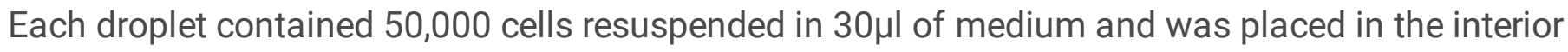
surface of the lid of a $55 \mathrm{~cm}^{2}$ Petri dish (ThermoFisher Scientific, USA) while the dish was filled with $7 \mathrm{ml}$ of sterile PBS to avoid the droplet to dry. After the deposition of the droplets in the dish's lid, it was turned upside down on the dish and placed overnight inside the incubator at $37^{\circ} \mathrm{C}$ in a humidified atmosphere of $5 \% \mathrm{CO}_{2}$. Then, the droplets were transferred into a 24 wells-ultra low binding-plate (ThermoFisher 
Scientific, USA) with the chondrogenic differentiation medium. The medium was replaced every 3 days for 21 days. Alcian blue staining assessed the differentiation. The photos were captured with an inverted optical microscope (Leica DMI3000B/Nikon Digital Camera Dxm1200F).

\section{Functional characterization of pUVEC}

Finally, to evaluate the functional activity of pUVEC, the cells were cultured in 8-wells chamber slides (Nunc Lab-Tek II Chamber Slide, ThermoFisher Scientific, USA) at 30,000 cells/well with an expansion medium incubated at $37^{\circ} \mathrm{C}$ in a humidified atmosphere of $5 \% \mathrm{CO}_{2}$ for two days. Then, the cells were treated with a Dil-Ac-LDL staining kit (Cell Applications, USA), following the manufacturer's instructions. The photos were captured with a fluorescence microscope (Leica DMI3000B/Nikon Digital Camera Dxm1200F) and processed using ImageJ 1.51s software.

\section{Abbreviations}

AF488: Alexa Fluor 488; AF546: Alexa Fluor 546; ALP: Alkaline Phosphatase; APC: Allophycocyanine; BSA: Bovine Serum Albumin; Dil-Ac-LDL: 1'-dioctadecyl-3,3,3',3'-tetramethyl-indocarbocyanine perchlorate Acetylated Low-Density Lipoprotein; DMEM: Dulbecco's Modified Eagle's Medium; EC: Endothelial Cells; ECM: Extracellular Matrix; EDTA: Ethylenediaminetetraacetic acid; FBS: Fetal Bovine Serum; FC: Flow Cytometry; FITC: Fluorescein isothiocyanate; MNC: Mononuclear Cells; MSC: Mesenchymal Stem/Stromal Cells; PBS: Phosphate Buffered Saline; PE: Phycoerythrin; PerCP-Cy5.5: Peridinin Chlorophyll Protein Cyanine 5.5; P/S: Penicillin/Streptomycin; pASMC: Porcine Aortic Smooth Muscle Cells; pBM-MSC: Porcine Bone Marrow derived-Mesenchymal Stem/Stromal Cells; pEGM: Porcine Endothelial Growth Medium; pUVEC: Porcine Umbilical Vein Endothelial Cells; RM: Regenerative Medicine; $\alpha$ SMA: alpha Smooth Muscle Actin; SM22 $\alpha$ : Smooth Muscle 22 alpha; SMC: Smooth Muscle Cells.

\section{Declarations}

\section{Ethics approval and consent}

All animals were housed at the Experimental Surgery Department under approved conditions and the tissues were harvested after intraoperative approved euthanasia. The experiments were performed in accordance with the European and ARRIVE guidelines regarding the protection of animals and were approved by the Animal Experimentation Committee of the Government of Aragón, Spain (ref. PI08/17).

\section{Competing interests' statement}

The authors declare that they have no competing interests.

\section{Funding}

Funding received by iBB-Institute for Bioengineering and Biosciences from FCT-Portuguese Foundation for Science and Technology (FCT) (UIDB/04565/2020) and FCT through the project PTDC/EQU- 
EQU/31651/2017. SM acknowledges FCT for the PhD fellowship PD/BD/114057/2015. PMB was supported by the PI15/00563 and PI18/00529 Research Project from Instituto de Salud Carlos III, Madrid, Spain.

\section{Authors' contributions}

SM carried out all the experiments, analyzed the results of the experiments, prepared the figures, and drafted the manuscript. IPP, PSMA and NSR isolated porcine endothelial cells and assisted with the cell culture experiments. MVF, OM, and AR have carried out the control care and handling of the animals used in this work and the surgical procedures used to obtain the porcine fetuses. LMS and SGA helped in the draft of the manuscript. MLBM and EOA provided help with all the immunofluorescence procedures performed. AFP participated in the study's design and coordination and in the analysis of the results of the experiments. PMB designed and coordinated the study, edited, revised, and accepted the final version of the manuscript. All authors read and approved the final manuscript.

\section{Acknowledgments}

SM thanks Cristiana Ulpiano for her help in the porcine endothelial cells' cultures and characterization, André Branco for his assistance in preparing flow cytometry graphical data and Maria Jesús Lozano for her help with cell culture experiments. SM also wishes to thank João Viana Gonçalinho and Ricardo Martins for their assistance editing of this manuscript.

\section{References}

1. Meng F, Assiri A, Dhar D, et al. Whole liver engineering: A promising approach to develop functional liver surrogates. Liver Int. 2017. p. 1759-1772.

2. Baptista PM, Siddiqui MM, Lozier G, et al. The use of whole organ decellularization for the generation of a vascularized liver organoid. Hepatology. 2011;53:604-617.

3. Uygun BE, Soto-Gutierrez A, Yagi H, et al. Organ reengineering through development of a transplantable recellularized liver graft using decellularized liver matrix. Nat. Med. 2010;16:814-820.

4. Yagi H, Fukumitsu K, Fukuda K, et al. Human-scale whole-organ bioengineering for liver transplantation: A regenerative medicine approach. Cell Transplant. 2013;

5. Caralt M, Velasco E, Lanas A, et al. Liver bioengineering. Organogenesis [Internet]. 2014;10:250-259. Available from: http://www.tandfonline.com/doi/abs/10.4161/org.29892.

6. Badylak SF, Taylor D, Uygun K. Whole-organ tissue engineering: Decellularization and recellularization of three-dimensional matrix scaffolds. Annu. Rev. Biomed. Eng. 2011;13:27-53.

7. Jain RK, Au P, Tam J, et al. Engineering vascularized tissue. Nat. Biotechnol. 2005. p. 821-823.

8. Pellegata AF, Tedeschi AM, De Coppi P. Whole organ tissue vascularization: Engineering the tree to develop the fruits. Front. Bioeng. Biotechnol. 2018. 
9. Ding B Sen, Nolan DJ, Guo P, et al. Endothelial-derived angiocrine signals induce and sustain regenerative lung alveolarization. Cell. 2011;147:539-553.

10. Takebe T, Sekine K, Enomura M, et al. Vascularized and functional human liver from an iPSC-derived organ bud transplant. Nature. 2013;499:481-484.

11. Hu J, Srivastava K, Wieland $M$, et al. Endothelial cell-derived Angiopoietin-2 controls liver regeneration as a spatiotemporal rheostat. Science (80-. ). 2014;343:416-419.

12. Poulos MG, Crowley MJP, Gutkin MC, et al. Vascular platform to define hematopoietic stem cell factors and enhance regenerative hematopoiesis. Stem Cell Reports. 2015;5:881-894.

13. Ramasamy SK, Kusumbe AP, Adams RH. Regulation of tissue morphogenesis by endothelial cellderived signals. Trends Cell Biol. 2015. p. 148-157.

14. Ding B Sen, Nolan DJ, Butler JM, et al. Inductive angiocrine signals from sinusoidal endothelium are required for liver regeneration. Nature. 2010;468:310-315.

15. Rafii S, Butler JM, Ding B Sen. Angiocrine functions of organ-specific endothelial cells. Nature. 2016. p. 316-325.

16. Orlando G, Farney AC, Iskandar SS, et al. Production and implantation of renal extracellular matrix scaffolds from porcine kidneys as a platform for renal bioengineering investigations. Ann. Surg. 2012;256:363-370.

17. Ribitsch I, Baptista PM, Lange-Consiglio A, et al. Large Animal Models in Regenerative Medicine and Tissue Engineering: To Do or Not to Do. Front. Bioeng. Biotechnol. 2020.

18. Beigi F, Patel M, Morales-Garza MA, et al. Optimized method for isolating highly purified and functional porcine aortic endothelial and smooth muscle cells. J. Cell. Physiol. 2017;232:3139-3145.

19. Davis J, Crampton SP, Hughes CCW. Isolation of human umbilical vein endothelial cells (HUVEC). J. Vis. Exp. 2007;

20. Ringe J, Kaps C, Schmitt B, et al. Porcine mesenchymal stem cells: Induction of distinct mesenchymal cell lineages. Cell Tissue Res. 2002;307:321-327.

21. Noort WA, Oerlemans MIFJ, Rozemuller $\mathrm{H}$, et al. Human versus porcine mesenchymal stromal cells: Phenotype, differentiation potential, immunomodulation and cardiac improvement after transplantation. J. Cell. Mol. Med. 2012;16:1827-1839.

22. Sekiya I, Larson BL, Smith JR, et al. Expansion of Human Adult Stem Cells from Bone Marrow Stroma: Conditions that Maximize the Yields of Early Progenitors and Evaluate Their Quality. Stem Cells. 2002;20:530-541.

23. Smith JR, Pochampally R, Perry A, et al. Isolation of a Highly Clonogenic and Multipotential Subfraction of Adult Stem Cells from Bone Marrow Stroma. Stem Cells. 2004;22:823-831.

24. Haasters F, Prall WC, Anz D, et al. Morphological and immunocytochemical characteristics indicate the yield of early progenitors and represent a quality control for human mesenchymal stem cell culturing. J. Anat. 2009;214:759-767. 
25. Rensen SSM, Doevendans PAFM, Van Eys GJJM. Regulation and characteristics of vascular smooth muscle cell phenotypic diversity. Netherlands Hear. J. 2007. p. 100-108.

26. Post ICJH, Weenink RP, Van Wijk ACWA, et al. Characterization and quantification of porcine circulating endothelial cells. Xenotransplantation. 2013;20:18-26.

27. Helmke BP, Thakker DB, Goldman RD, et al. Spatiotemporal analysis of flow-induced intermediate filament displacement in living endothelial cells. Biophys. J. 2001;80:184-194.

28. Conway DE, Breckenridge MT, Hinde E, et al. Fluid shear stress on endothelial cells modulates mechanical tension across VE-cadherin and PECAM-1. Curr. Biol. 2013;23:1024-1030.

29. Voyta JC, Via DP, Butterfield CE, et al. Identification and isolation of endothelial cells based on their increased uptake of acetylated-low density lipoprotein. J. Cell Biol. 1984;99:2034-2040.

30. Ott HC, Matthiesen TS, Goh SK, et al. Perfusion-decellularized matrix: Using nature's platform to engineer a bioartificial heart. Nat. Med. 2008;14:213-221.

31. Maghsoudlou P, Georgiades F, Smith H, et al. Optimization of liver decellularization maintains extracellular matrix micro-architecture and composition predisposing to effective cell seeding. PLoS One. 2016;11.

32. Cleaver O, Melton DA. Endothelial signaling during development. Nat. Med. 2003. p. 661-668.

33. Shaheen MF, Joo DJ, Ross JJ, et al. Sustained perfusion of revascularized bioengineered livers heterotopically transplanted into immunosuppressed pigs. Nat. Biomed. Eng. 2019;

34. Bosch P, Pratt SL, Stice SL. Isolation, characterization, gene modification, and nuclear reprogramming of porcine mesenchymal stem cells. Biol. Reprod. 2006;74:46-57.

35. Rho GJ, Kumar BM, Balasubramanian S. Porcine mesenchymal stem cells - Current technological status and future perspective. Front. Biosci. 2009;14:3942-3961.

36. Arrizabalaga JH, Nollert MU. Properties of porcine adipose-derived stem cells and their applications in preclinical models. Adipocyte. 2017. p. 217-223.

37. AA T, AV K, AA S. Mesenchymal stem cells from the domestic ungulates: trends and outliers. Int. J. Vet. Sci. Res. 2018;023-031.

38. Pittenger MF, Mackay AM, Beck SC, et al. Multilineage potential of adult human mesenchymal stem cells. Science (80-. ). 1999;284:143-147.

39. Kolf CM, Cho E, Tuan RS. Mesenchymal stromal cells. Biology of adult mesenchymal stem cells: Regulation of niche, self-renewal and differentiation. Arthritis Res. Ther. 2007.

40. Ock SA, Baregundi Subbarao R, Lee YM, et al. Comparison of immunomodulation properties of porcine mesenchymal stromal/stem cells derived from the bone marrow, adipose tissue, and dermal skin tissue. Stem Cells Int. 2016;2016.

41. Kumar BM, Jin HF, Kim JG, et al. Differential gene expression patterns in porcine nuclear transfer embryos reconstructed with fetal fibroblasts and mesenchymal stem cells. Dev. Dyn. 2007;236:435446. 
42. Lim SY, Kim YS, Ahn Y, et al. The effects of mesenchymal stem cells transduced with Akt in a porcine myocardial infarction model. Cardiovasc. Res. 2006;70:530-542.

43. Brückner S, Tautenhahn HM, Winkler S, et al. A fat option for the pig: Hepatocytic differentiated mesenchymal stem cells for translational research. Exp. Cell Res. 2014;321:267-275.

44. Schwartz SM. Smooth muscle migration in atherosclerosis and restenosis. J. Clin. Invest. 1997. p. 2814-2817.

45. Schwartz SM. The intima a new soil. Circ. Res. 1999. p. 877-879.

46. Adams LD, Geary RL, McManus B, et al. A comparison of aorta and vena cava medial message expression by cDNA array analysis identifies a set of 68 consistently differentially expressed genes, all in aortic media. Circ. Res. 2000;87:623-631.

47. Hao H, Gabbiani G, Bochaton-Piallat ML. Arterial smooth muscle cell heterogeneity: Implications for atherosclerosis and restenosis development. Arterioscler. Thromb. Vasc. Biol. 2003. p. 1510-1520.

48. Chamley-Campbell J, Campbell GR, Ross R. The smooth muscle cell in culture. Physiol. Rev. 1979. p. $1-61$.

49. Battula VL, Treml S, Bareiss PM, et al. Isolation of functionally distinct mesenchymal stem cell subsets using antibodies against CD56, CD271, and mesenchymal stem cell antigen-1. Haematologica. 2009;94.

50. Bühring HJ, Treml S, Cerabona F, et al. Phenotypic characterization of distinct human bone marrowderived MSC subsets. Ann. N. Y. Acad. Sci. 2009.

51. Rossignoli F, Caselli A, Grisendi G, et al. Isolation, characterization, and transduction of endometrial decidual tissue multipotent mesenchymal stromal/stem cells from menstrual blood. Biomed Res. Int. 2013;2013.

52. Holifield B, Helgason T, Jemelka S, et al. Differentiated vascular myocytes: Are they involved in neointimal formation? J. Clin. Invest. 1996;97:814-825.

53. Frid MG, Aldashev AA, Dempsey EC, et al. Smooth muscle cells isolated from discrete compartments of the mature vascular media exhibit unique phenotypes and distinct growth capabilities. Circ. Res. 1997;81:940-952.

54. Hao H, Ropraz P, Verin V, et al. Heterogeneity of smooth muscle cell populations cultured from pig coronary artery. Arterioscler. Thromb. Vasc. Biol. 2002;22:1093-1099.

55. Ponta H, Sherman L, Herrlich PA. CD44: From adhesion molecules to signalling regulators. Nat. Rev. Mol. Cell Biol. 2003. p. 33-45.

56. Lesley J, Hyman R, Kincade PW. CD44 and Its Interaction with Extracellular Matrix. Adv. Immunol. 1993;

57. Zaniboni A, Bernardini C, Alessandri M, et al. Cells derived from porcine aorta tunica media show mesenchymal stromallike cell properties in in vitro culture. Am. J. Physiol. - Cell Physiol. 2014;306.

58. Jain M, He Q, Lee W Sen, et al. Role of CD44 in the reaction of vascular smooth muscle cells to arterial wall injury. J. Clin. Invest. 1996;97:596-603. 
59. Skinner MP, Raines EW, Ross R. Dynamic expression of alpha 1 beta 1 and alpha 2 beta 1 integrin receptors by human vascular smooth muscle cells. Alpha 2 beta 1 integrin is required for chemotaxis across type I collagen-coated membranes. Am. J. Pathol. [Internet]. 1994;145:1070-1081. Available from: https://europepmc.org/articles/PMC1887428.

60. Jones $\mathrm{JI}$, Prevette T, Gockerman A, et al. Ligand occupancy of the alpha-V-beta3 integrin is necessary for smooth muscle cells to migrate in response to insulin-like growth factor. Proc. Natl. Acad. Sci. U. S. A. [Internet]. 1996;93:2482-2487. Available from: https://pubmed.ncbi.nlm.nih.gov/8637900.

61. Annussek T, Szuwart T, Kleinheinz J, et al. In vitro inhibition of HUVECs by low dose methotrexate insights into oral adverse events. Head Face Med. 2014;10.

62. Chrusciel M, Bodek G, Kirtiklis L, et al. Immortalization of swine umbilical vein endothelial cells (SUVECs) with the simian virus 40 large-T antigen. Mol. Reprod. Dev. 2011;78:597-610.

63. Burciaga-Nava JA, Reyes-Romero MA, Avelar-González FJ, et al. Establishment and characterization of porcine aortic endothelial cell cultures with prolonged replicative lifespan by a non-enzymatic method. Vitr. Cell. Dev. Biol. - Anim. 2009;45:15-18.

64. Hätinen OPA, Lähteenvuo JE, Korpela $\mathrm{HJ}$, et al. Isolation of fresh endothelial cells from porcine heart for cardiovascular studies: A new fast protocol suitable for genomic, transcriptomic and cell biology studies. BMC Mol. Cell Biol. 2019;20.

65. Smith AG, Cheu J, Presnell SC. Phenotypic and Functional Characterization of human Umbilical Vein Endothelial Cells (hUVEC) Cultures Established under Physiologic vs. Atmospheric Oxygen Tension. FASEB J. 2020;34:1-1.

66. Päll T, Pink A, Kasak L, et al. Soluble CD44 interacts with intermediate filament protein vimentin on endothelial cell surface. PLoS One. 2011;6.

\section{Figures}




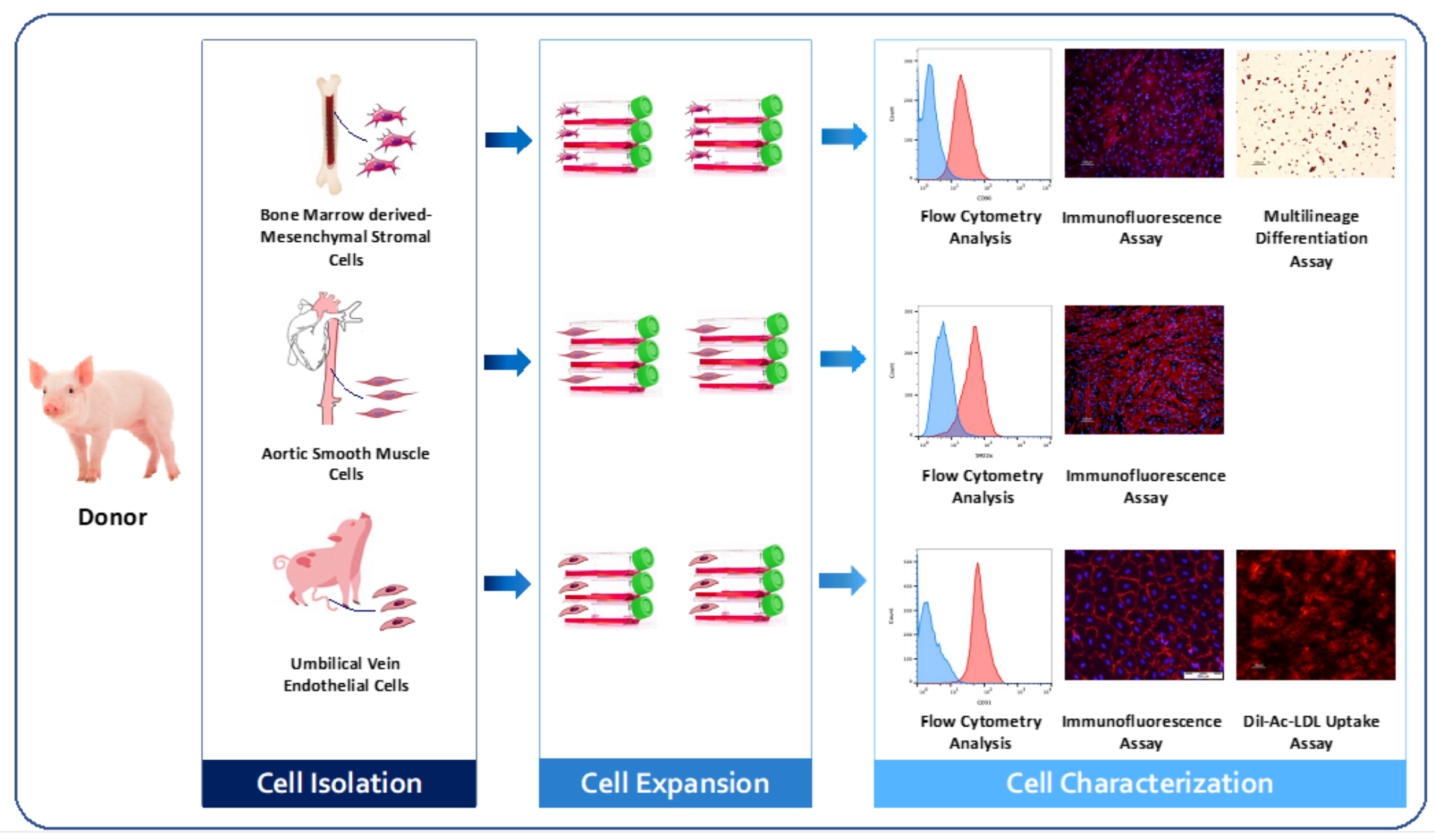

\section{Figure 1}

Flow chart for parallel cell isolation, expansion and characterization of single-donor porcine primary vascular cells for bioengineering and other research applications. This procedure integrates separate knowledge and elicits the parallel isolation, expansion and subsequent characterization of three distinctive primary cell phenotypes from a single porcine donor.
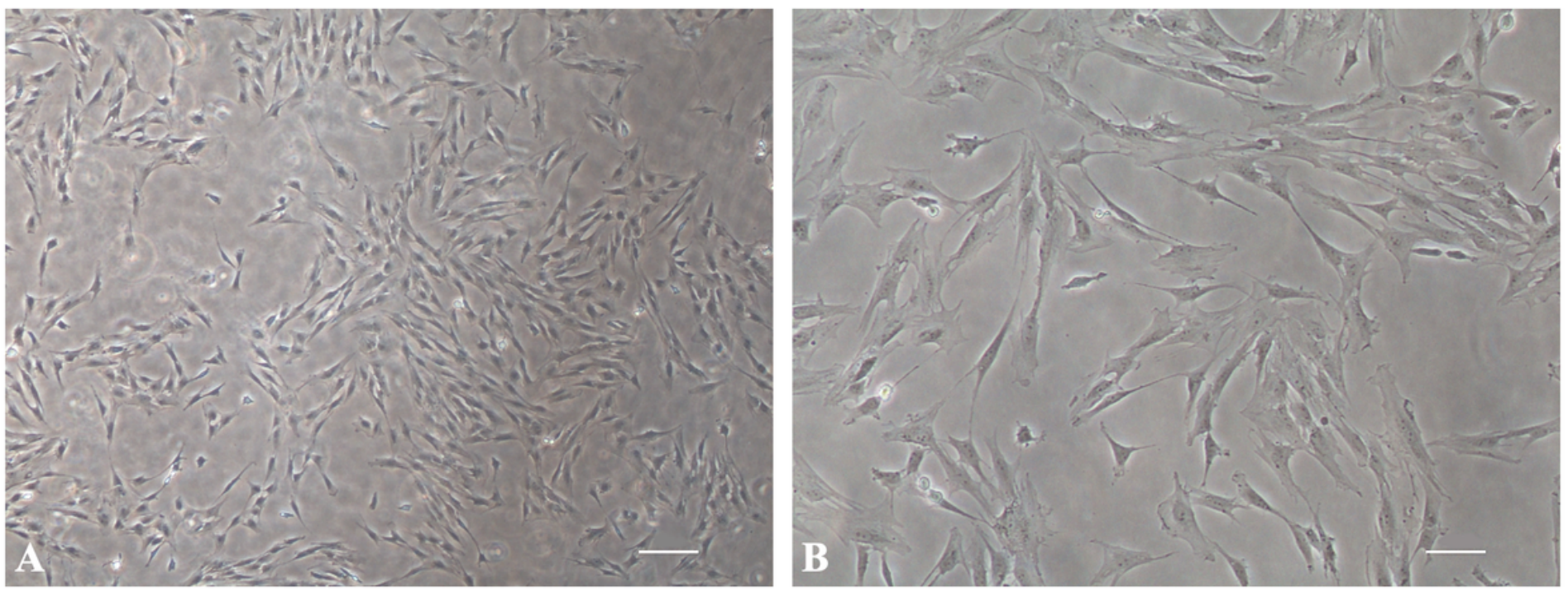

Figure 2 
Microscopic images of pBM-MSC. A) Early passage pBM-MSC (passage 1). Scale bar: $200 \mu \mathrm{m}$. B) Heterogeneous culture containing different populations of pBM-MSC (passage 2). Scale bar: $100 \mu \mathrm{m}$.

A
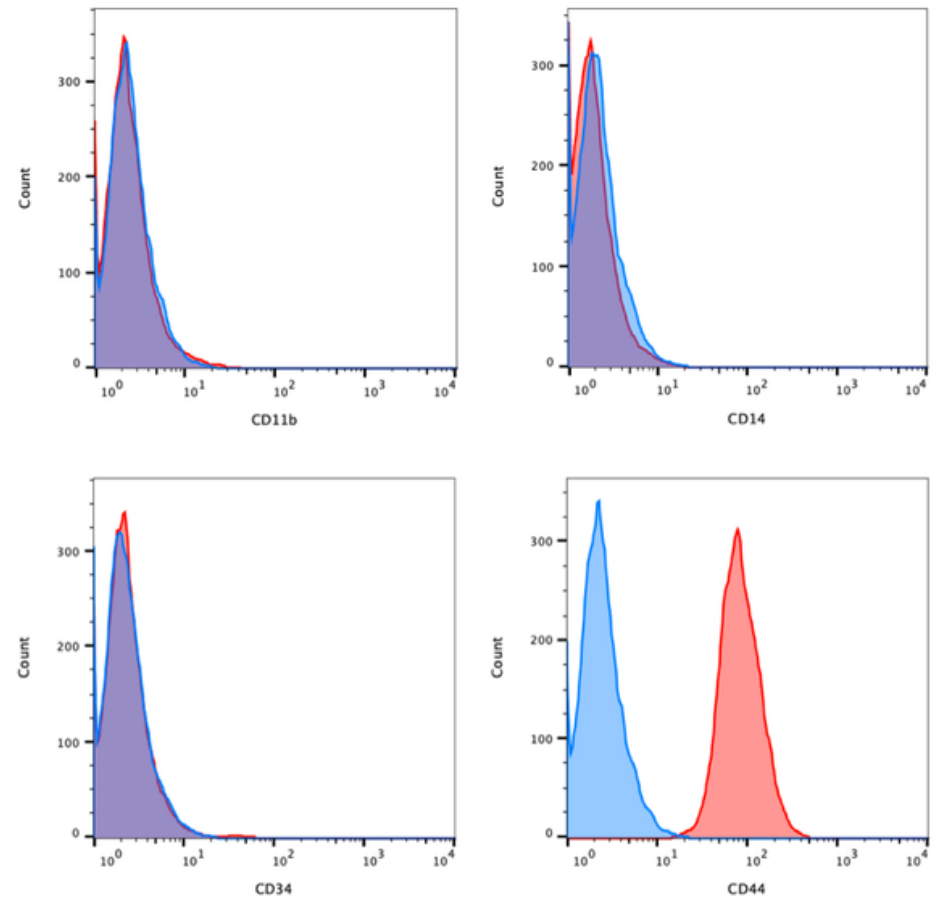
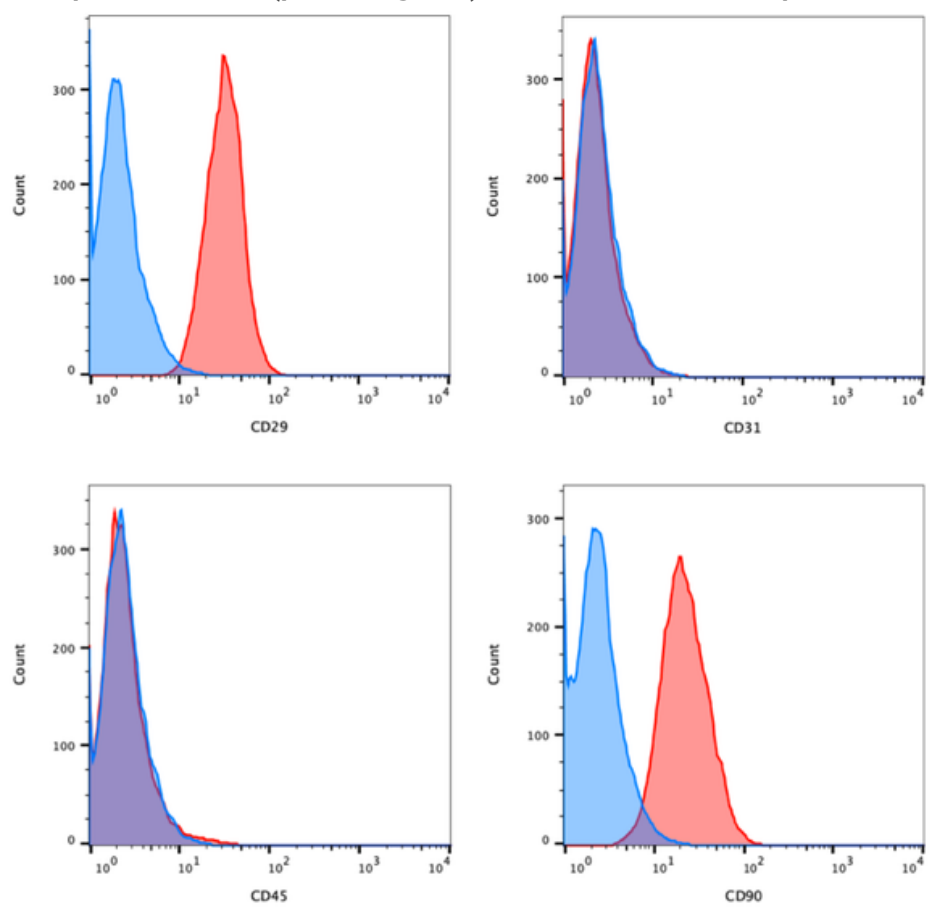

B
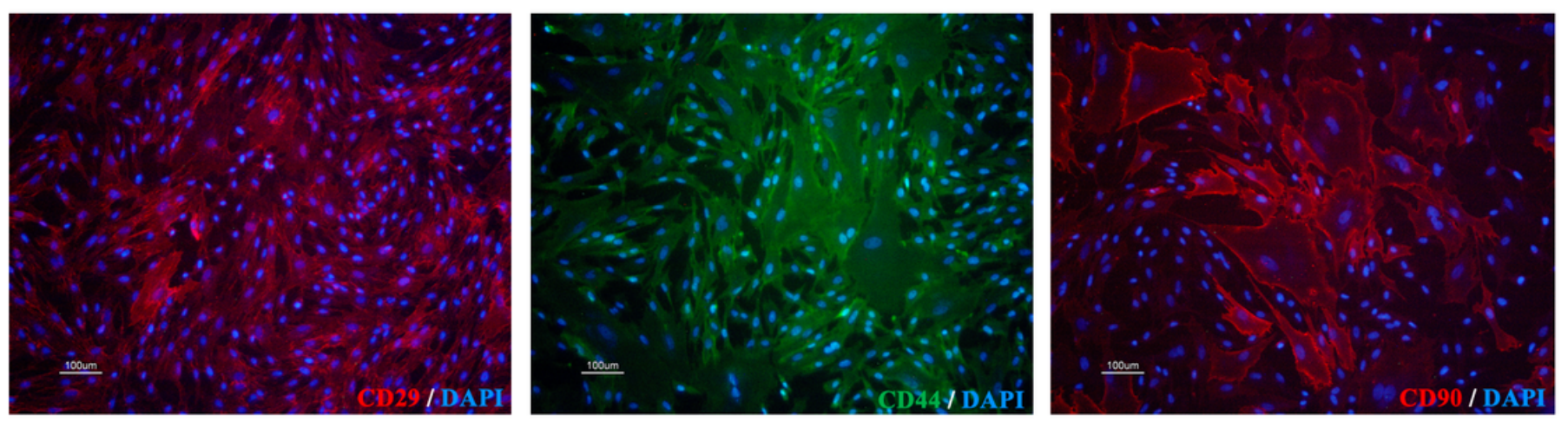

Figure 3

Phenotypical characterization of bone marrow-derived pBM-MSC. A) Flow cytometry analysis for a set of surface markers showing expression of CD29, CD44, and CD90. B) Immunofluorescence analysis for a panel of MSC surface markers (CD29, CD44, CD90), showing positive expression. Scale bar: $100 \mu \mathrm{m}$. 
Adipogenic Differentiation
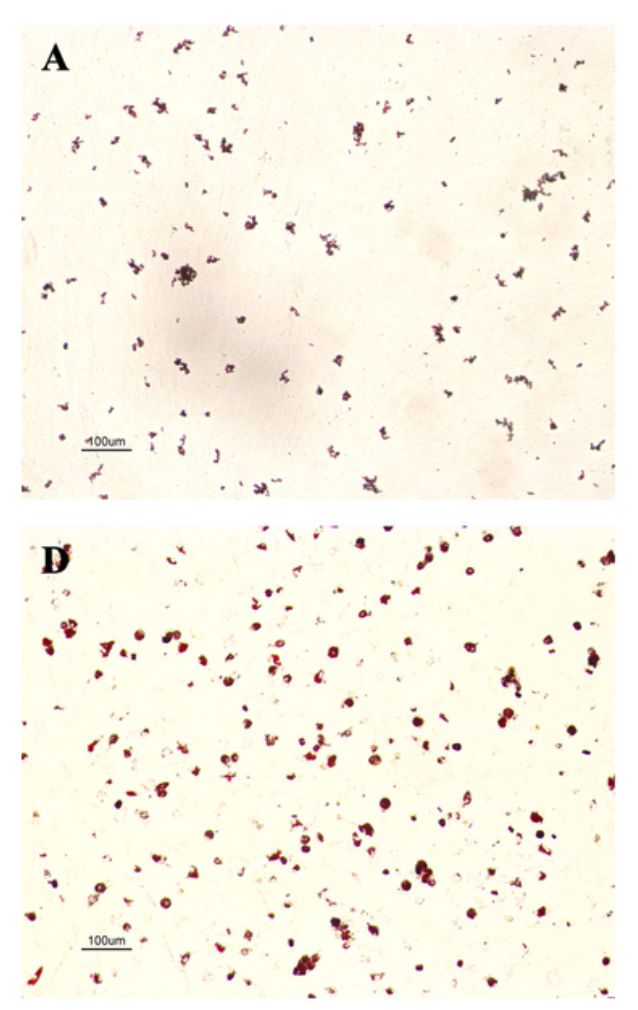

\section{Osteogenic Differentiation}
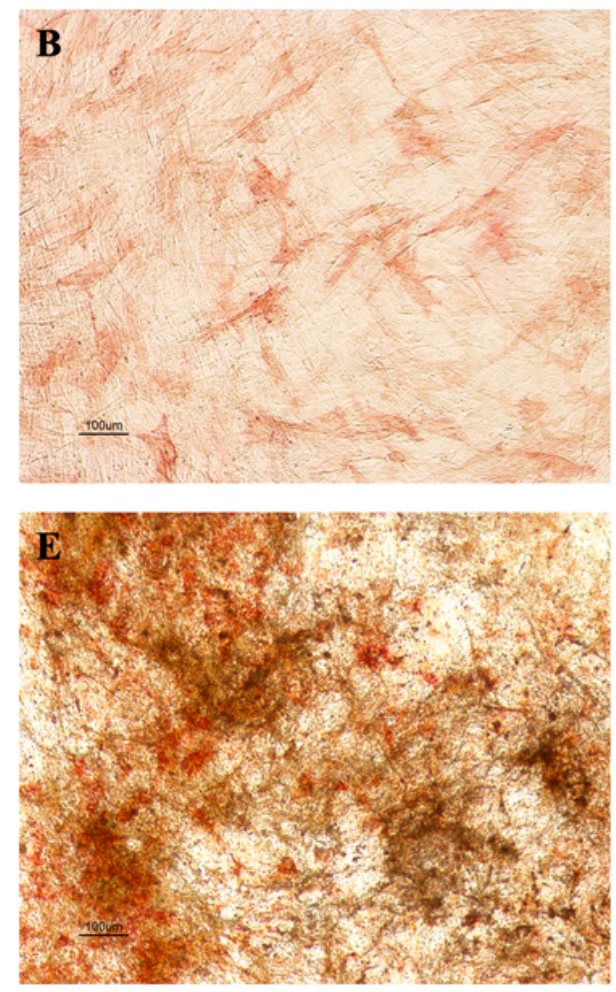

\section{Chondrogenic Differentiation}

$\mathbf{C}$

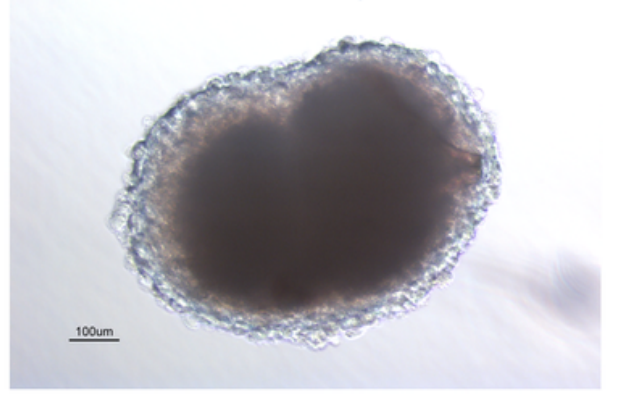

F

\section{Figure 4}

Functional characterization of pBM-MSC by trilineage differentiation assay. Scale bar: $100 \mu \mathrm{m}$. A) Negative control for adipogenesis stained with Oil Red O. B) Negative control for osteogenesis stained with ALP/silver nitrate. C) Negative control for chondrogenesis stained with Alcian Blue. D) Cells cultured with adipogenesis differentiation medium stained positive with Oil Red O. E) Cells cultured with osteogenesis
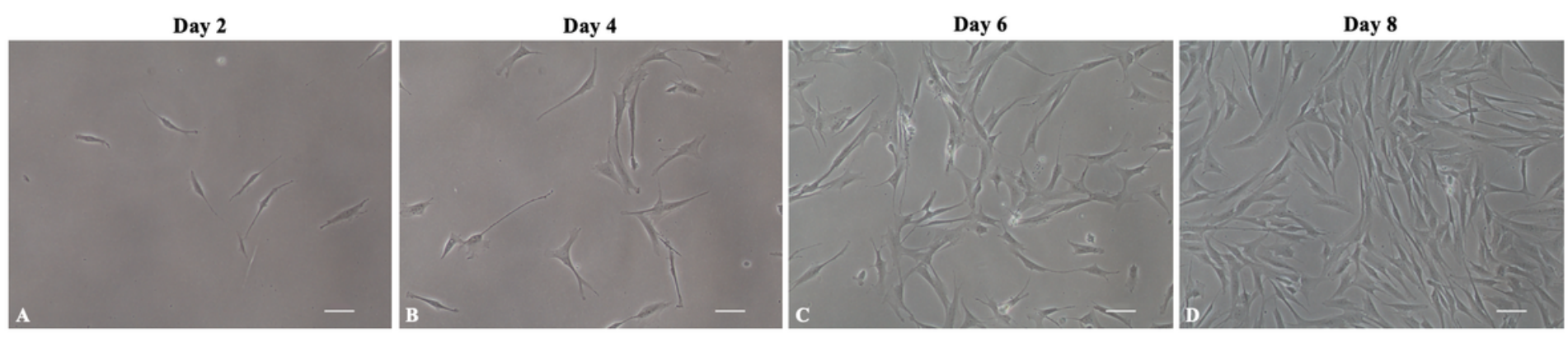

\section{Figure 5}

Microscopic images of pASMC. Scale bar: $100 \mu \mathrm{m}$. Morphology of pASMC cultured with DMEM/FBS at days $2(A), 4(B), 6(C)$, and $8(D)$ after the initial seeding. 
A
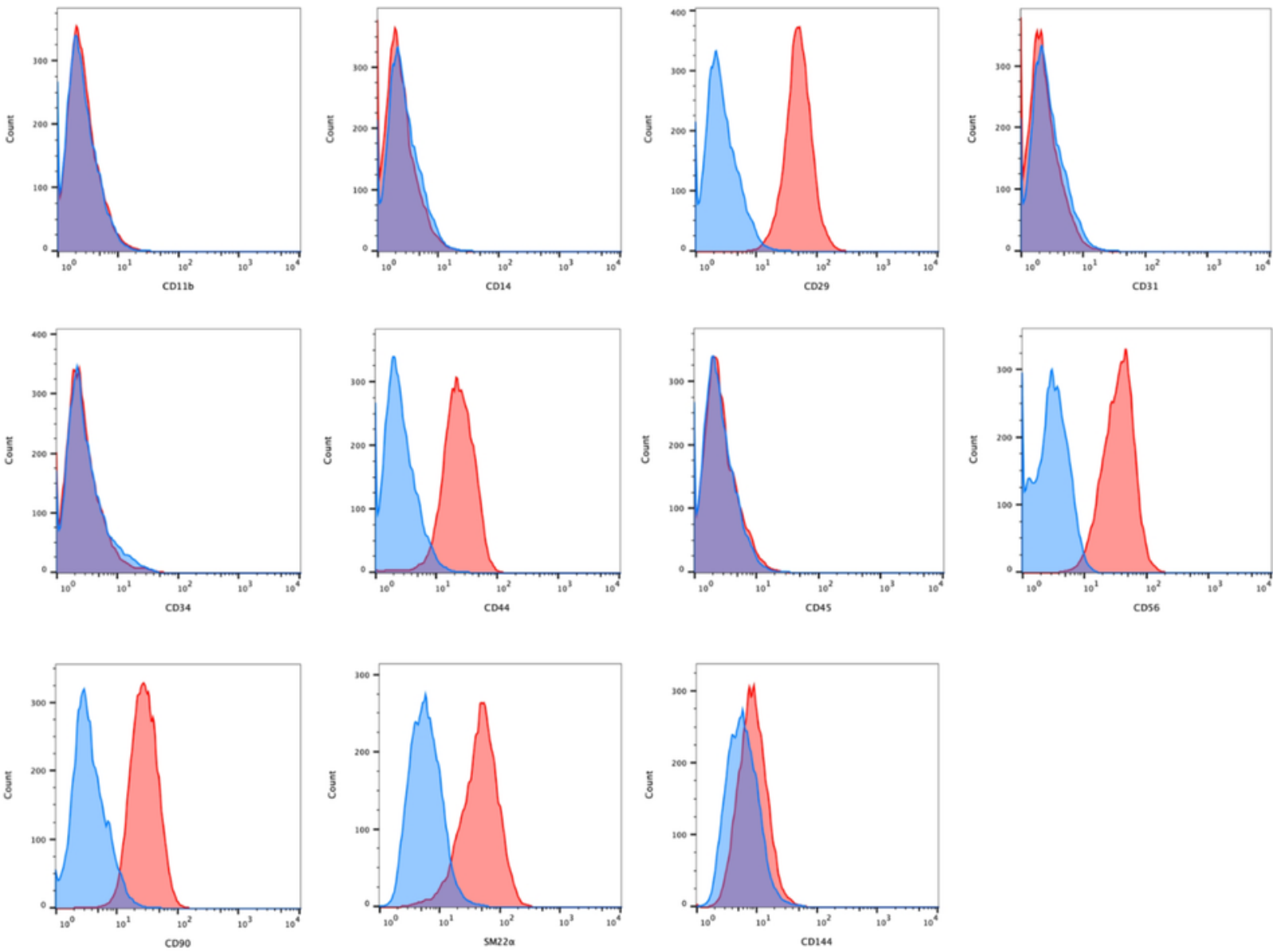

B
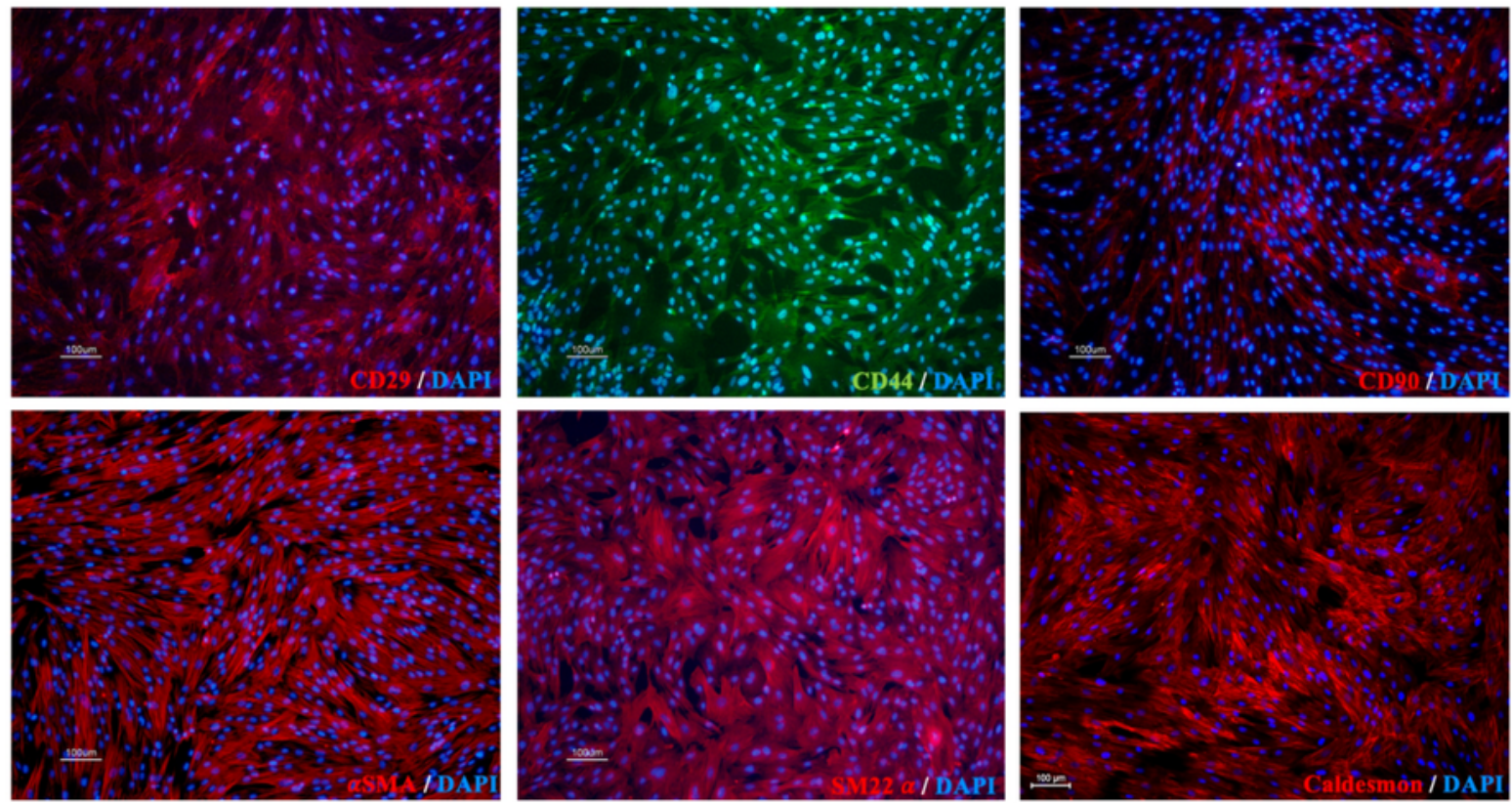

\section{Figure 6}

Phenotypical characterization of pASMC. A) Flow cytometry analysis for extracellular and intracellular markers of SMC isolated from porcine aorta showing positive staining for CD29, CD44, Cd56, CD90, and SM22 $\alpha$. B) Immunofluorescence analysis for extracellular and intracellular markers of SMC (CD29, CD44, CD90, $\alpha$ SMA, SM22 $\alpha$, and Caldesmon) showing positive expression of these markers. Scale bar: $100 \mu \mathrm{m}$. 


\section{Before Sorting}

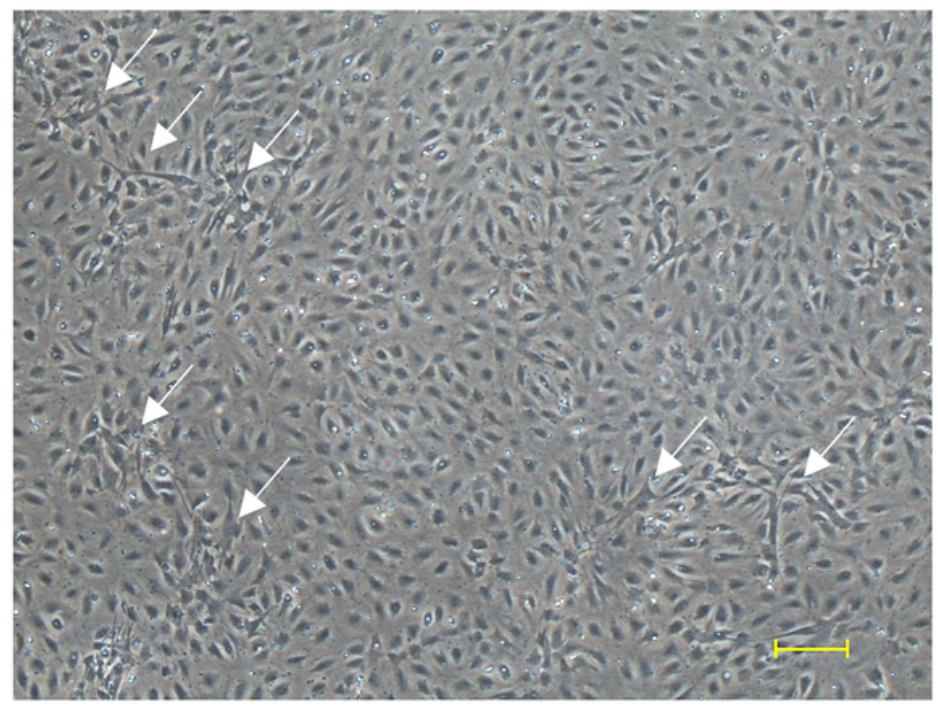

After Sorting

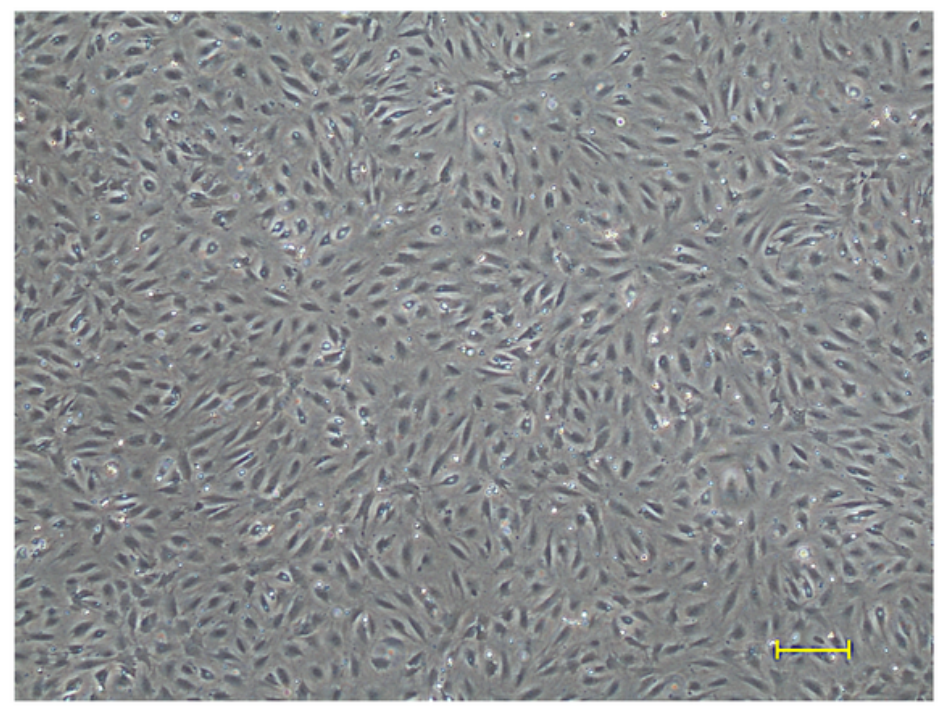

Figure 7

Microscopic images of pUVEC isolated from porcine umbilical vein. The cells show the characteristic cobblestone phenotype when confluent in culture. The arrows indicate the contaminating cells in the culture. Scale bar: $100 \mu \mathrm{m}$. 
$\mathbf{A}$
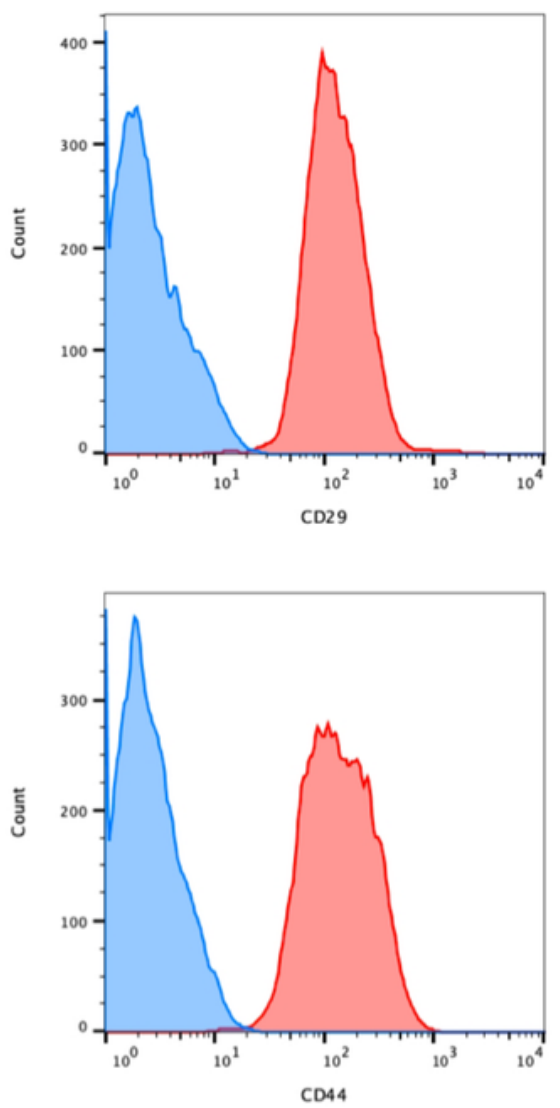

B
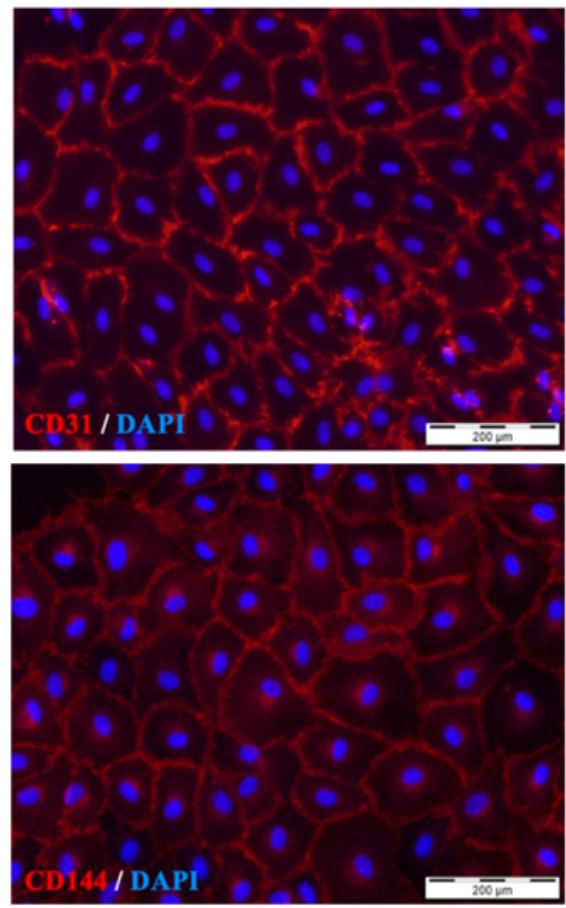
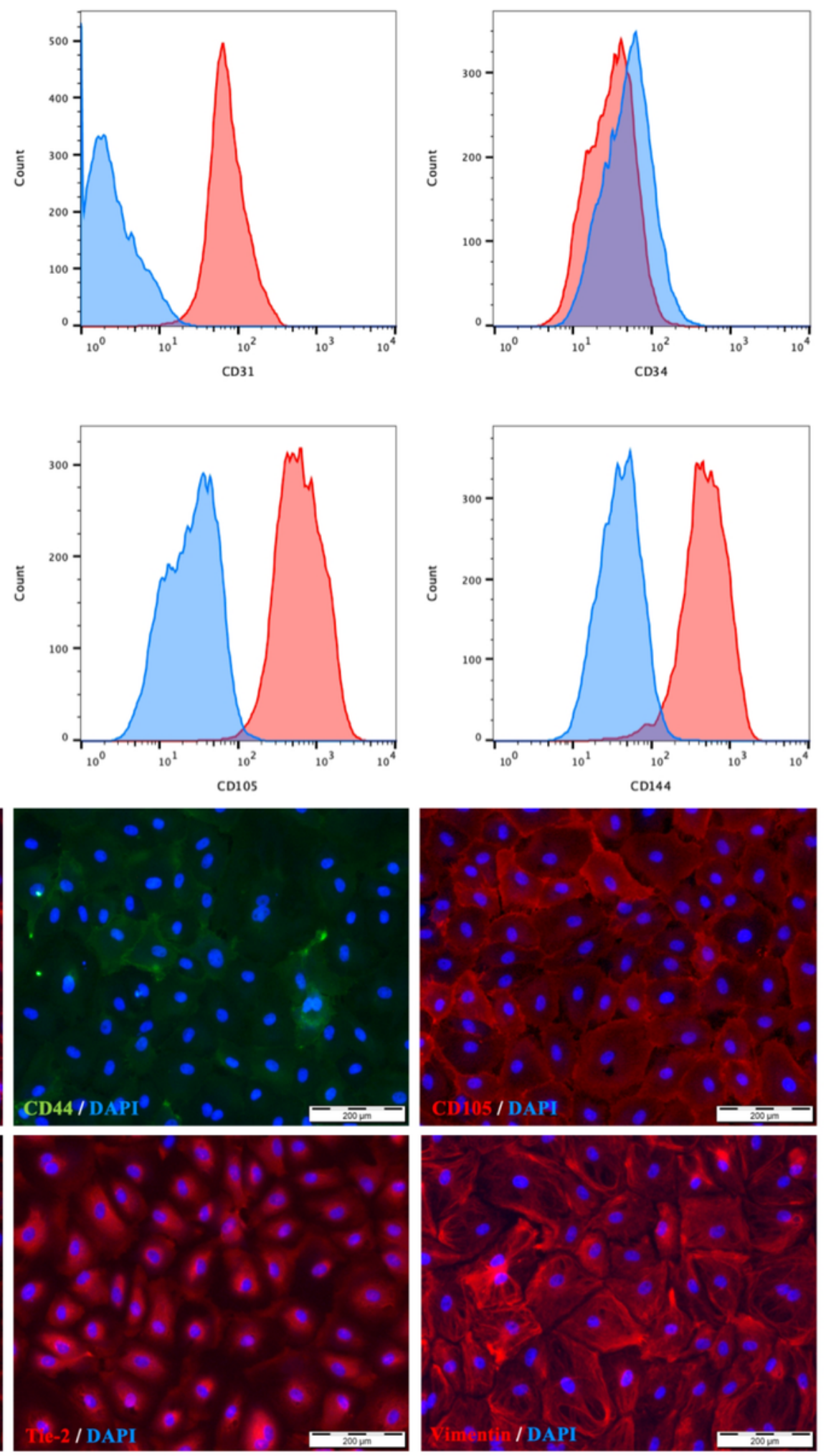

\section{Figure 8}

Immunophenotypical characterization of pUVEC. A) Flow cytometry analysis for extracellular and intracellular markers of pUVEC isolated from porcine umbilical vein showed robust expression of CD29, CD31, CD44, CD105, and CD144. B) Immunofluorescence analysis for extracellular and intracellular markers of EC (CD31, CD44, CD105, CD144, Tie-2, and Vimentin) was also positive. Scale bar: $200 \mu \mathrm{m}$. 


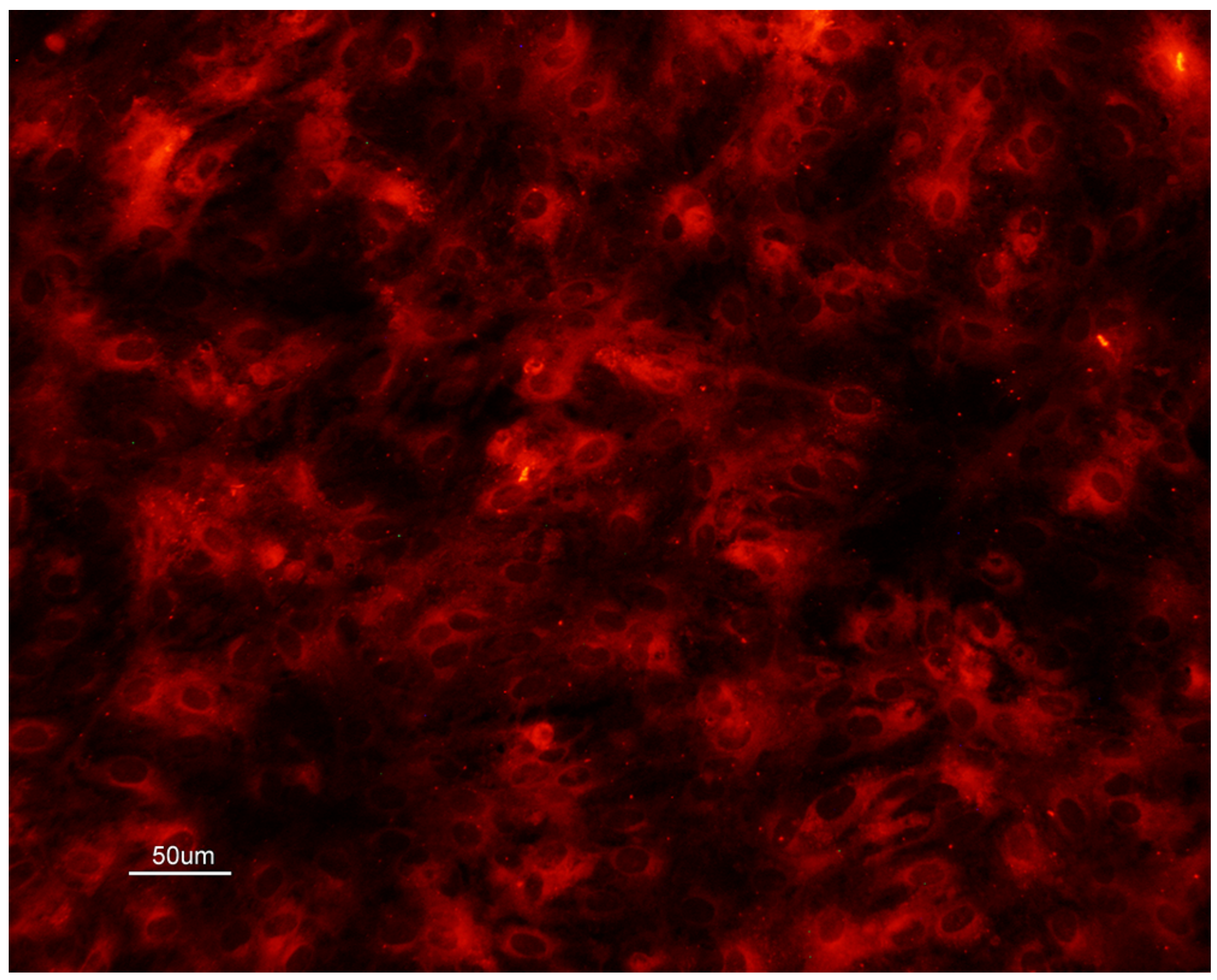

\section{Figure 9}

Dil-Ac-LDL uptake assay of pUVEC cultured under static conditions with pEGM culture medium. The cells that internalized Dil-Ac-LDL are shown as red cells. Scale bar: $50 \mu \mathrm{m}$. 